\title{
Chaotropes trigger conformational rearrangements differently in Concanavalin A
}

\author{
SHREYASI ASTHANA $^{\mathrm{a}}$, SUJIT KUMAR BHUTIA ${ }^{\mathrm{a}}$, HAREKRUSHNA SAHOO ${ }^{\mathrm{b}}$ \\ and SUMAN JHA ${ }^{\mathrm{a}, *}$ (D) \\ ${ }^{a}$ Department of Life Science, National Institute of Technology Rourkela, Odisha 769 008, India \\ ${ }^{b}$ Department of Chemistry, National Institute of Technology Rourkela, Odisha 769 008, India \\ E-mail: jhas@ nitrkl.ac.in
}

MS received 12 March 2017; revised 9 May 2017; accepted 1 June 2017

\begin{abstract}
Concanavalin A (ConA) is a plant lectin having industrial and biological applications. Concanavalin A changes conformation upon exposure to different stress conditions, like exposure to sodium dodecyl sulphate, guanidine hydrochloride, varying hydronium ion potential, etc. The conformational changes were studied using circular dichroism spectroscopy and the structural rigidity of ConA was explored using fluorescence spectroscopy, taking tryptophan as an intrinsic and 8-anilino-1-naphthalenesulfonic acid as an extrinsic fluorescence probes, in different stress conditions. ConA loses the quaternary structure in all the studied stress conditions, which further leads to denaturation of the protein. However, the mechanism of denaturation varied with the studied conditions, like different SDS concentrations and hydronium ion potentials, wherein the protein undergoes a conformational rearrangement from $\beta$-sheet to $\alpha$-helix. Moreover, $\mathrm{GdnHCl}$ triggered complete denaturation of ConA into a predominantly random coil conformation. The results suggested that denaturation of ConA follows different pathways depending on the chemical properties and concentrations of the denaturants used.
\end{abstract}

Keywords. Concanavalin A; jelly roll motif; sodium dodecyl sulphate; guanidine hydrochloride; molten globule conformation.

\section{Introduction}

The three-dimensional structure of any native protein is determined by "thermodynamic principle", which means that amino acid sequence and environmental physico-chemical parameters lead the sequence into three-dimensional structure with lowest free energy content. ${ }^{1}$ The folding of a protein into a compact threedimensional structure is the most fundamental aspect of biological self-assembly. Studying the conformation of a protein in different conditions provides useful insights for determining its stability and biological activity. Tracking the protein conformational changes can help in identifying optimal conditions to preserve functionality, stability and broaden the range of applications. ${ }^{2}$ The balance between activity and stability is, therefore, necessary.

\footnotetext{
*For correspondence
}

Conformational studies of proteins are usually done in different physical and chemical conditions. For unfolding studies, chaotropes like guanidine hydrochloride $(\mathrm{GdnHCl})$ and urea are used. ${ }^{3} \mathrm{GdnHCl}$ is believed to be an ideal chemical denaturant for protein unfolding reaction. Interaction of $\mathrm{GdnHCl}$ occurs to both folded and unfolded states of proteins, but the binding affinity and the number of binding sites for each of the states are different. The precise mechanism, however, is not understood properly. ${ }^{4}$ On the contrary, reports relating to the denaturation of proteins by detergents have been few. Ionic detergents can denature proteins by binding strongly to opposite charge and hydrophobic side chains at millimolar concentrations. All $\alpha$-helical proteins are sensitive towards sodium dodecyl sulfate (SDS) denaturation, but all $\beta$-sheet proteins are suggested to be more resistant to the denaturation by SDS. ${ }^{5}$ Besides chaotropes, the physico-chemical environment like the extremes of temperature and hydronium ion concentration can also induce conformational changes in protein.

Electronic supplementary material: The online version of this article (doi:10.1007/s12039-017-1333-z) contains supplementary material, which is available to authorized users. 
Heat denaturation, on the other hand, interferes with intra-molecular interactions in a globular protein, by enhancing the internal energy of the residues and by reducing the surface tension of cage water. ${ }^{6}$ Similarly, change in $\mathrm{pH}$ can also have profound effects on stability and activity of a protein.

Lectins are a family of carbohydrate-binding all- $\beta$ proteins, mostly existing in multimeric forms. Monomeric subunits are similar in structure with huge variations in the respective quaternary structures. Therefore, lectins present an interesting model for studying conformational states in proteins because of their conserved secondary and tertiary structures. Studying multimeric proteins will provide important insights into the impact of inter- and intra- molecular interactions on stability of the proteins. ConA is a plant lectin predominantly extracted from Canavalia ensiformis (jack bean). The monomer is composed of 237 amino acids with molecular weight of $26 \mathrm{kDa}$. In physiological conditions, the protein exists as a tetramer. ${ }^{7}$ It is an all- $\beta$ protein, containing three $\beta$ sheets in the form of jelly roll motif. ${ }^{8}$ First, a 6-stranded back sheet, second, a 7-stranded front sheet and third, a 5-stranded smaller sheet that forms a roof between the two large sheets and holds them together, ${ }^{9}$ sterically hindering two hydrophobic cores from exposure to hydrophilic environment (Figure 1). These $\beta$-sheets are connected by several turns of varying sizes. ${ }^{10}$ The first hydrophobic core is present between the front and back sheet, and the second hydrophobic core is present between the front sheet and a loop, conserved for carbohydrate binding. ${ }^{11}$ Two identical dimers have been shown to associate with the centre of their back sheets forming a tetramer. ${ }^{9}$ It has high specificity for sugars like glucose and mannose, and one binding site each for transition metals, typically $\mathrm{Ca}^{2+}$ and $\mathrm{Mn}^{2+}$. Demetallizaion of ConA results in the loss of carbohydrate binding. The amino acid residues involved in interaction with $\mathrm{Mn}^{2+}$ and $\mathrm{Ca}^{2+}$ ions are Glu8, Tyr12, Asn14, Asp19 and His24. The sugar binding pocket of ConA is surrounded by Asn14, Asp16, Asp208, Tyr12, Tyr100, Leu99 and Arg228. The number of tryptophan residue in a protein is very crucial as far as the biophysical studies are concerned, since they act as reporters of microenvironment of the protein. Hence, tracing changes in intrinsic property of tryptophan directly correlate with the effects of additives on protein conformation. Each subunit of ConA has four tryptophan residues, (Trp40/88/109/182). In monomeric ConA, Trp40/109 remain buried and are present close to the residues involved in $\mathrm{Ca}^{2+}$ ion binding, while Trp88/182 remain exposed. On other hand, Trp88/182 residues of each subunit are part of monomermonomer interface in dimer (Figure 1). Thus, all the

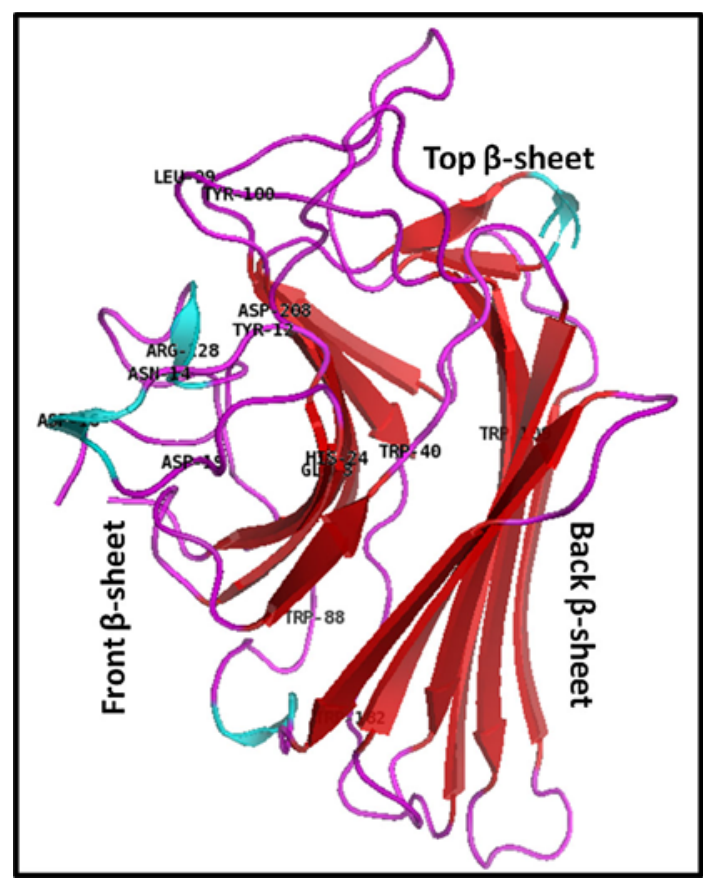

Figure 1. Jelly roll motif represented in monomeric ConA, depicting front, top and back $\beta$-sheets arrangement.

Trp residues in native ConA either remain buried in hydrophobic core or are present at the loci of interfacial interactions, making all four residues non-accessible to solvent. ${ }^{7,12}$

The stability of oligomeric proteins like ConA greatly depends on their subunit interactions. Inter-subunit interactions and buried surface area between the subunits contribute to a higher stability to oligomeric proteins in comparison to monomeric proteins, which rely solely on intra-subunit interactions for their stability. The impact of subunit interactions on stability of lectins have been studied in many lectins including ConA. ${ }^{13,14}$ A solvent-dependent ConA and winged bean acidic agglutinin (WBA) denaturation study correlated the inter-subunit interactions with stability of lectin proteins. The work showed that different oligomerization patterns and intersubunit interactions affect stability of the protein, ConA was more stable than WBA. ${ }^{13}$ However, soybean agglutinin (SBA) showed relatively stable quaternary conformation compared to ConA. The enhanced stability was attributed to four additional salt bridges in SBA compared to ConA, hence imparting SBA a greater stability. ${ }^{15}$ Lectin proteins, especially ConA, have been used in isolation of plasma membrane proteins. ${ }^{16}$ It has several biological functions like induction of autophagy in cancer cells, agglutination of various cell types, affinity purification of polysaccharides and glycoproteins of biological importance, etc. ${ }^{17-19}$ Keeping in mind the applications of lectins, the 
protein has been studied for its folding and unfolding mechanism. However, the conformational dynamics of ConA in presence of different chaotropes remain yet to be elucidated. Hence, the present study on the relative conformational changes in ConA upon transferring the protein from native condition to stress conditions like varying concentrations of hydronium ions, SDS and $\mathrm{GdnHCl}$. The study indicated that the conformational transition adopted by the protein upon change in chemical environment is entirely different.

\section{Experimental}

\subsection{Materials}

Jack bean (Canavalia ensiformis) seeds were a gift from Dr. Sujit Kumar Bhutia, Department of Life Science, N.I.T Rourkela, Odisha, India. Sephadex-G-100, GdnHCl, SDS, and 8-anilino-1-napthalenesulfonic acid (ANS) were obtained from Sigma (USA). All the other reagents used were of analytical grade. All the buffers and solutions were prepared in MilliQ water, and they were filtered through $0.45 \mu \mathrm{m}$ syringe filter before spectrophotometric measurements. Molar concentration of ANS was determined spectrophotometrically using the extinction coefficient of $7800 \mathrm{M}^{-1} \mathrm{~cm}^{-1}$ at 372 $\mathrm{nm} \cdot{ }^{20}$ All the experiments were performed in $10 \mathrm{mM}$ phos-

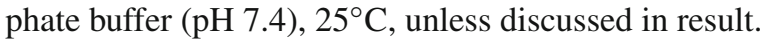

\subsection{Protein purification}

Native ConA was purified using the protocol of Goldstein and Agarwal with slight modifications. ${ }^{21}$ Briefly, $10 \% \mathrm{w} / \mathrm{v}$ jackbean meal (washed, dried and ground powder) was prepared in $10 \mathrm{mM}$ phosphate buffer and stirred overnight at $4^{\circ} \mathrm{C}$. Next morning, the mixture was centrifuged at $7000 \mathrm{rpm}$ and the supernatant was subjected to salting out process using sequential 30 and $90 \%$ ammonium sulfate precipitaion. The pellet obtained after each precipitation was re-dissolved in phosphate buffer, and dialysed against phosphate buffer to remove salts. The dialysed solution was solubilised in phosphate buffer, and centrifuged at $7000 \mathrm{rpm}, 30 \mathrm{mins}$ at $4^{\circ} \mathrm{C}$. The clear supernatant obtained was further purified using Sephadex-G-100 as an affinity matrix, and ConA was eluted with $0.2 \mathrm{M}$ glucose. The eluted samples were again dialysed to remove glucose. The purity of the protein was confirmed by sodium dodecyl sulfate polyacrylamide gel electrophoresis (SDS-PAGE) and native PAGE. The concentration of ConA was determined spectrophotometrically by Bradford assay. ${ }^{22}$ Pure fractions were lyophilized and stored at $4{ }^{\circ} \mathrm{C}$, for further use. Protein stock solution was prepared in filtered and degassed $10 \mathrm{mM}$ phosphate buffer.

\subsection{Spectroscopic measurements}

Absorption spectra were recorded on a UV-Visible spectrophotometer (Agilent Technologies, Cary100, Singapore) in a quartz cuvette. Fourier transform infrared (FT-IR) spectroscope serves as a useful tool for qualitative analysis of secondary structures in proteins. ${ }^{23}$ The IR spectra of native protein was recorded on attenuated total reflection fourier transform infrared (ATR-FTIR) spectroscope, Bruker, Germany, in a range of $500-2000 \mathrm{~cm}^{-1}$ with a resolution of 2 $\mathrm{cm}^{-1}$, and the data was further processed from the region $1580-1710 \mathrm{~cm}^{-1}$ specific for amide I peaks in proteins. ${ }^{23}$ Tryptophan residues in ConA served as intrinsic fluorescent probes, for following the structural perturbations by denaturants. ${ }^{24}$ Intrinsic fluorescence measurements were performed at $2 \mu \mathrm{M}$ ConA using LS-55 Perkin Elmer spectrofluorimeter in a $10 \mathrm{~mm}$ cuvette, with slit set at $5 \mathrm{~nm}$ each for excitation and emission with a scan speed $100 \mathrm{~nm} / \mathrm{min}$. The emission spectra were recorded between $300-400 \mathrm{~nm}$ with an excitation wavelength of $280 \mathrm{~nm}$. Circular Dichroism (CD) presents one of the most efficient techniques in probing secondary structures in proteins. ${ }^{25} \mathrm{CD}$ spectroscopic measurements were performed in far UV range (190-260 nm), for $10 \mu \mathrm{M}$ of ConA in $1 \mathrm{~mm}$ cuvette using JASCO- J1500 CD spectropolarimeter, purged with $\mathrm{N}_{2}$ gas, and equipped with a Jasco Peltier-type temperature controller system.

\subsection{8-Anilinonaphthalene-1-sulfonate (ANS) binding}

Extrinsic fluorescence dyes also play important roles in studying protein conformations. ${ }^{26}$ The surface hydrophobicity changes in a protein can be probed by ANS. It is an extensively utilized fluorescence probe for characterization of accessible hydrophobic patches in proteins. It has also been used to study conformational changes and molten globule structures formed upon protein unfolding. ${ }^{27}$ The dye on binding to proteins shows a blue shift in fluorescence emission maximum, accompanied with an increase in fluorescence intensity and lifetime. The change is attributed to hydrophobicity of a binding site and the restricted mobility of ANS within the site. ${ }^{28}$

ANS binding experiments were performed using a Synergy H1 multimode plate-reader (Biotek, USA). ANS was excited at $388 \mathrm{~nm}$ and the emission spectra were recorded from 420 $\mathrm{nm}$ to $580 \mathrm{~nm}$ with a $5 \mathrm{~nm}$ step size at $25^{\circ} \mathrm{C}$. For each set of experiments with or without the denaturant, the protein and ANS concentrations used were 5 and $100 \mu \mathrm{M}$, respectively.

\subsection{Protein denaturation}

For $\mathrm{pH}$ and chemical mediated denaturation, intrinsic tryptophan fluorescence, ANS fluorescence and CD spectra were recorded. Stock solutions of the chemical denaturants, $\mathrm{GdnHCl}$ and SDS, were prepared in $10 \mathrm{mM}$ phosphate buffer $\mathrm{pH} 7.4$, and filtered through $0.45 \mu \mathrm{m}$ membrane filter. Buffers of different $\mathrm{pH}$, ranging from 2 to 11 , were prepared with either the acidic or the basic component of phosphate buffer in combination with $\mathrm{HCl}$ or $\mathrm{NaOH}$ depending upon required $\mathrm{pH}$. For each denaturation experiment, constant protein concentration was incubated with varying denaturant concentrations, keeping the final reaction volumes constant, i.e., $0.5 \mathrm{~mL}$ each. The mixtures were then incubated at $25^{\circ} \mathrm{C}$ for $4-6 \mathrm{~h}$ to ensure 
that the equilibrium was achieved. For extrinsic fluorescence experiments, ANS was added to a final concentration of 100 $\mu \mathrm{M}$, and incubated at room temperature in dark for $30 \mathrm{mins}$ for ANS binding.

\section{Results and Discussion}

\subsection{Native Concanavalin A}

SDS-PAGE showed a major band at $28 \mathrm{KDa}$, corresponding to the weight of its monomeric subunit. Native PAGE also yielded a single band on the gel, which further confirmed the purity of the protein. Secondary structure attributes of native Con A was further confirmed by its peculiar motif signal, jelly roll motif, using FT-IR and CD spectroscopy. Circular Dichroism spectra of purified protein indicated a trough at $222 \pm 2 \mathrm{~nm}$ and a positive peak at $195 \mathrm{~nm}$, a characterstic CD spectra for lectin like proteins. Hence, the purified protein was confirmed as ConA (Figure 2B). ${ }^{29,30}$ Additionally, the FT-IR spectrum of native ConA showed a major peak at $1636 \mathrm{~cm}^{-1}$, corresponding to native $\beta$-sheets in proteins (Figure 2A); the crystal structure of ConA has been previously shown to contain predominantly $\beta$-sheets. ${ }^{31}$ Other important peaks were also observed at 1658 and $1696 \mathrm{~cm}^{-1}$, corresponding to turns that connect stranded sheets to each other. ${ }^{32,33}$

\section{2 pH induced conformational changes}

The conformational changes in ConA in response to different $\mathrm{pH}$ were first followed by $\mathrm{CD}$ spectropolarimeter, since the protein has a very distinct $\mathrm{CD}$ spectrum. ConA shows a characteristic peak at $223 \mathrm{~nm}$, at pH 6 and 7 .
ConA exists as a dimer at $\mathrm{pH} 6$ and as a tetramer at $\mathrm{pH}$ 7. ${ }^{34}$ At both $\mathrm{pH}$, it is seen that the prevalent structure, jelly roll motif, is retained with an insignificant change in the peak position. A single negative peak at $223 \mathrm{~nm}$ is observed for both $\mathrm{pH}$, only differing in magnitude. At $\mathrm{pH} 2$ there is a slight shift to lower wavelength (221 $\mathrm{nm}$ ), which also accounts for $\beta$-sheets in proteins. The observation is consistent with previous study. ${ }^{34}$ Lower $\mathrm{pH}(<4)$ leads into ConA demetallization, which results in exposure of amino acids involved in interaction with metal ions. ${ }^{35}$ Hence, the demetallization slightly opens the conformation of ConA at lower $\mathrm{pH}$. However, overall change in secondary structure remains insignificant. ${ }^{35}$ At basic $\mathrm{pH}, 9$ and 11, a significant shift in trough towards lower wavelength was seen with a pronounced increase in ellipticity. Major negative peaks at 216 and $209 \mathrm{~nm}$ were observed, indicating the presence of $\alpha$ helix-like secondary structure elements (Figure 3A). It was shown earlier that in $\mathrm{pH}$ range of $8-9$, ConA undergoes an irreversible conformational transition. ${ }^{30}$ Moreover, it was also shown that ConA forms amorphous aggregates at $\mathrm{pH} 8.9 .{ }^{36}$ This suggests that at $\mathrm{pH}$ $>8$, key conformational changes in the secondary structure of ConA leads to formation of some atypical, but relatively stable, conformational states.

Intrinsic fluorescence measurements prove a crucial tool when it comes to screen minor changes in physicochemical environment around the probe. Tryptophan residues in proteins act as intrinsic fluorophore probe to track microenvironment polarity surrounding its indole ring. ${ }^{37}$ Native ConA at $\mathrm{pH} 7$ had emission maxima at $338 \mathrm{~nm}\left(\lambda_{\max }\right)$ upon excitation at $280 \mathrm{~nm}$ whereas at $\mathrm{pH}$ 6 , the dimer showed an insignificant change in the intensity and emission maxima. Likewise, for $\mathrm{pH} 2$ and 3 , the
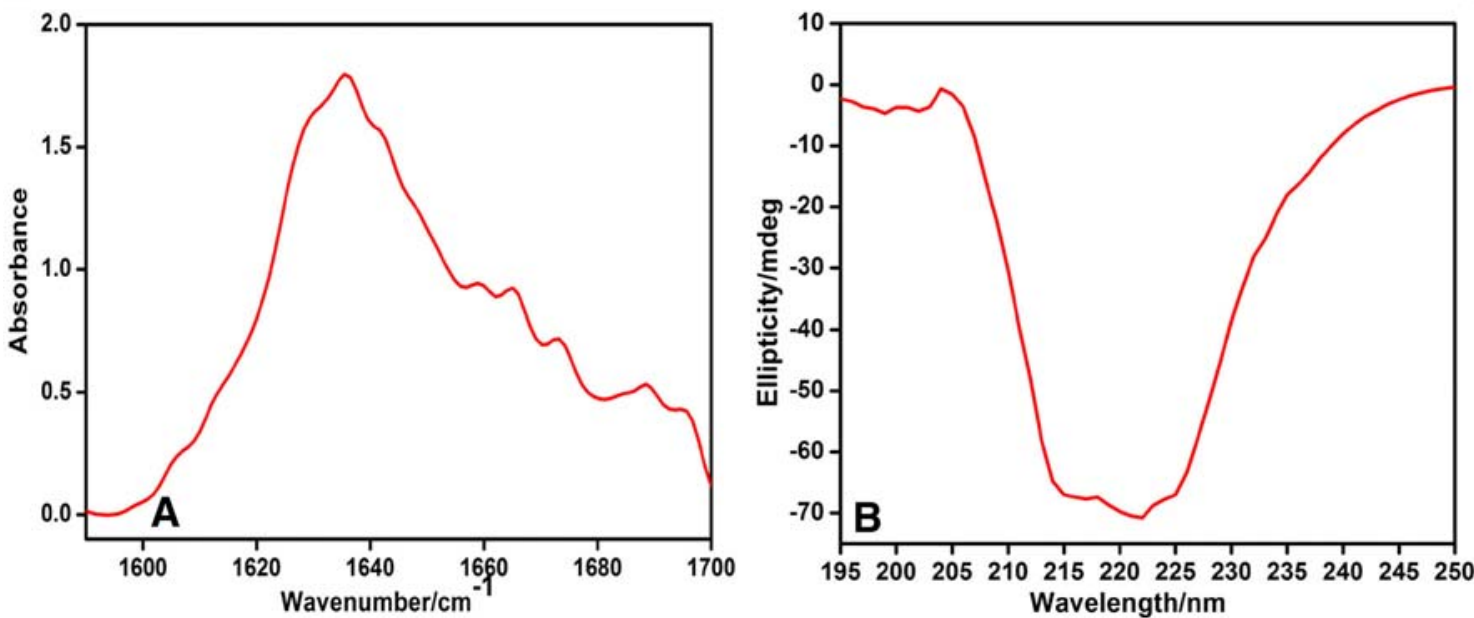

Figure 2. Secondary structure characteristics of native ConA. (A) FT-IR spectrum of $10 \mu \mathrm{M}$ purified ConA, showing a major peak at $1636 \mathrm{~cm}^{-1}$, and (B) far UV-CD spectrum of $10 \mu \mathrm{M}$ native ConA showing ellipticity minimum at $223 \mathrm{~nm}$, a characteristic lectin-like ellipticity. 

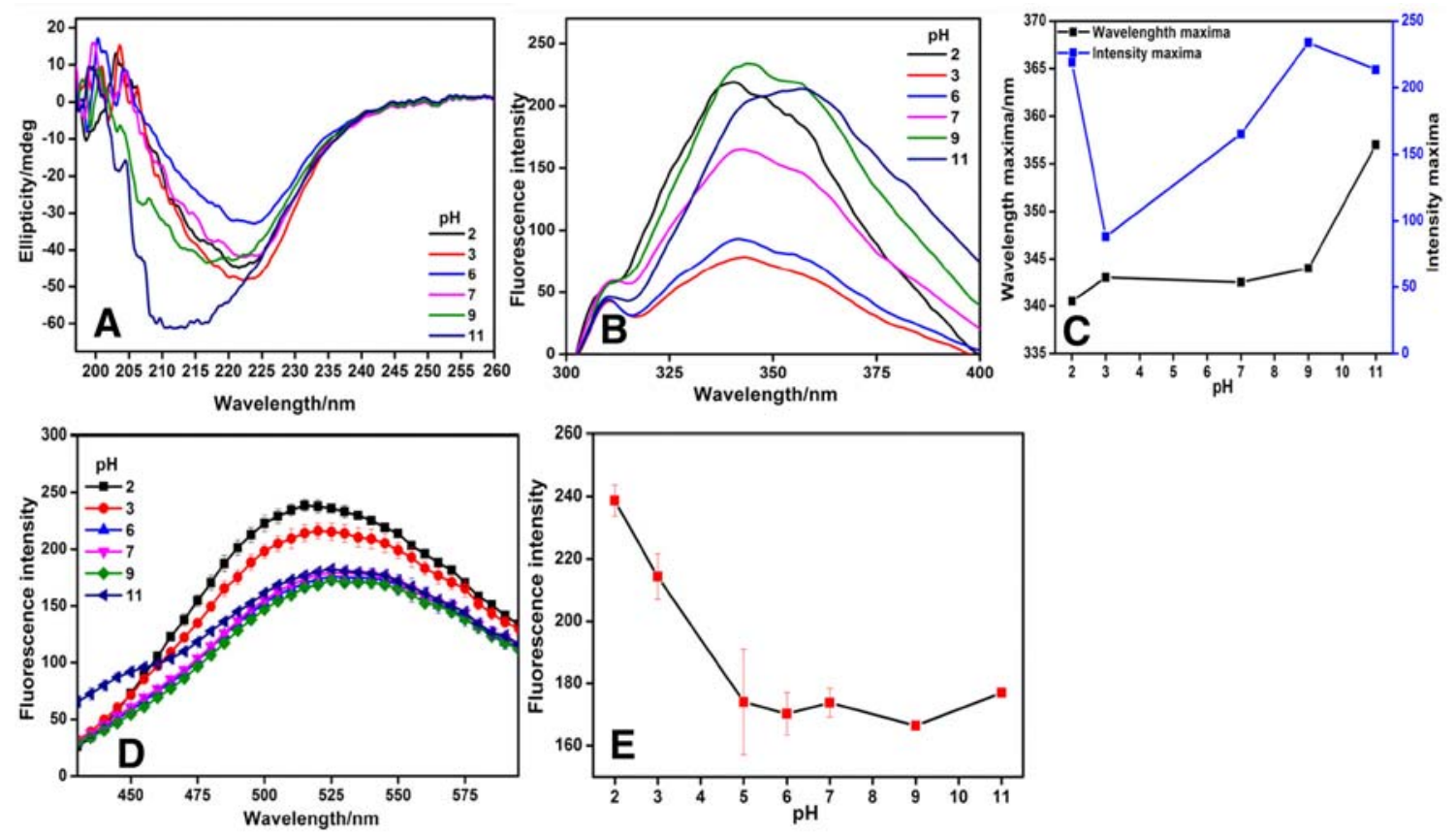

Figure 3. Conformational changes in ConA at different $\mathrm{pH}$. (A) far-UV CD spectra from $195 \mathrm{~nm}$ to 260 $\mathrm{nm}$ at different $\mathrm{pH}$ conditions; (B) tryptophan fluorescence emission spectra from $300 \mathrm{~nm}$ to $400 \mathrm{~nm}$ at different $\mathrm{pH}$ conditions; (C) intensity and wavelength maxima of tryptophan fluorescence plotted against $\mathrm{pH}$ values; (D) ANS fluorescence spectra from $420 \mathrm{~nm}$ to $580 \mathrm{~nm}$ upon binding to ConA at different $\mathrm{pH}$ values; and (E) change in ConA bound ANS fluorescence intensity plotted against $\mathrm{pH}$ values (each represented data is an average of three scans with scan speed of $100 \mathrm{~nm} / \mathrm{min}$ for both CD and fluorescence measurements).

$\lambda_{\max }$ for emission were at $341 \mathrm{~nm}$. However, there was a significant increase in intensity at $\mathrm{pH} 2$, which could be due exposure of Trp88/182 upon demetallization at $\mathrm{pH}$ 2. At alkaline $\mathrm{pH} 9$ and 11, the emission maxima further red shifted to 345 and $358 \mathrm{~nm}$, respectively (Figures $3 \mathrm{~B}$ and $3 \mathrm{C}$ ), which are accounted for exposure of the indole rings to polar environment upon ConA denaturation. Tryptophan emission maximum and intensity are shown to be influenced by the exposure to incident light, polarity of its micro-environment, hydrogen bond and other non-covalent interactions. A study indicated that there is a pronounced red shift in the wavelength maxima when the polarity of the tryptophan microenvironment increases. ${ }^{38}$

To study change in surface hydrophobicity of ConA with change in $\mathrm{pH}$, ANS was used which is specific for binding to hydrophobic patches in proteins. Native ConA at pH 7 showed a major peak around 525 $\mathrm{nm}$, revealing less number of accessible hydrophobic patches and more compact ConA conformation. At $\mathrm{pH}$ 2, upon binding to ConA, ANS emission maximum, $\lambda_{\max }$, is blue shifted from $525 \mathrm{~nm}$ to $515 \mathrm{~nm}$, with a significant increase in the quantum yield (Figures 3DE). The observation indicated that ConA demetallization at low $\mathrm{pH}$ results in increased numbers of accessible hydrophobic regions. Additionally, the presence of dimeric states at low $\mathrm{pH}$ suggests relatively more exposed hydrophobic patches than in the tetrameric form. At basic $\mathrm{pH}$ (pH 9 and 11), no significant changes in the ANS fluorescence spectra were observed. However, the CD spectra indicate significant change in the conformation. Hence, the $\mathrm{pH}$ data altogether indicated that ConA is a very stable protein for a broad range of $\mathrm{pH}$, while at extreme alkaline $\mathrm{pH}$, ConA deviates from its specific conformation to helices-like conformation with stable tertiary structure.

\subsection{SDS induced conformational changes}

SDS is an amphiphile, which has long been known to induce conformational changes in protein at a concentration of as low as $0.1 \mathrm{mM} .{ }^{39} \mathrm{SDS}$ induced perturbation in ConA structure was studied using tryptophan residues and ANS fluorescence in absence and presence of varying SDS concentration. Whereas, the change in secondary structure with varying SDS concentrations was studied using CD spectropolarimeter. In CD experiments, initially upon increasing SDS concentration to $1 \mathrm{mM}$, a significant increase in ellipticity was observed (Figure 4A) without any significant shift in the wavelength. The change depicts that the SDS binding to 

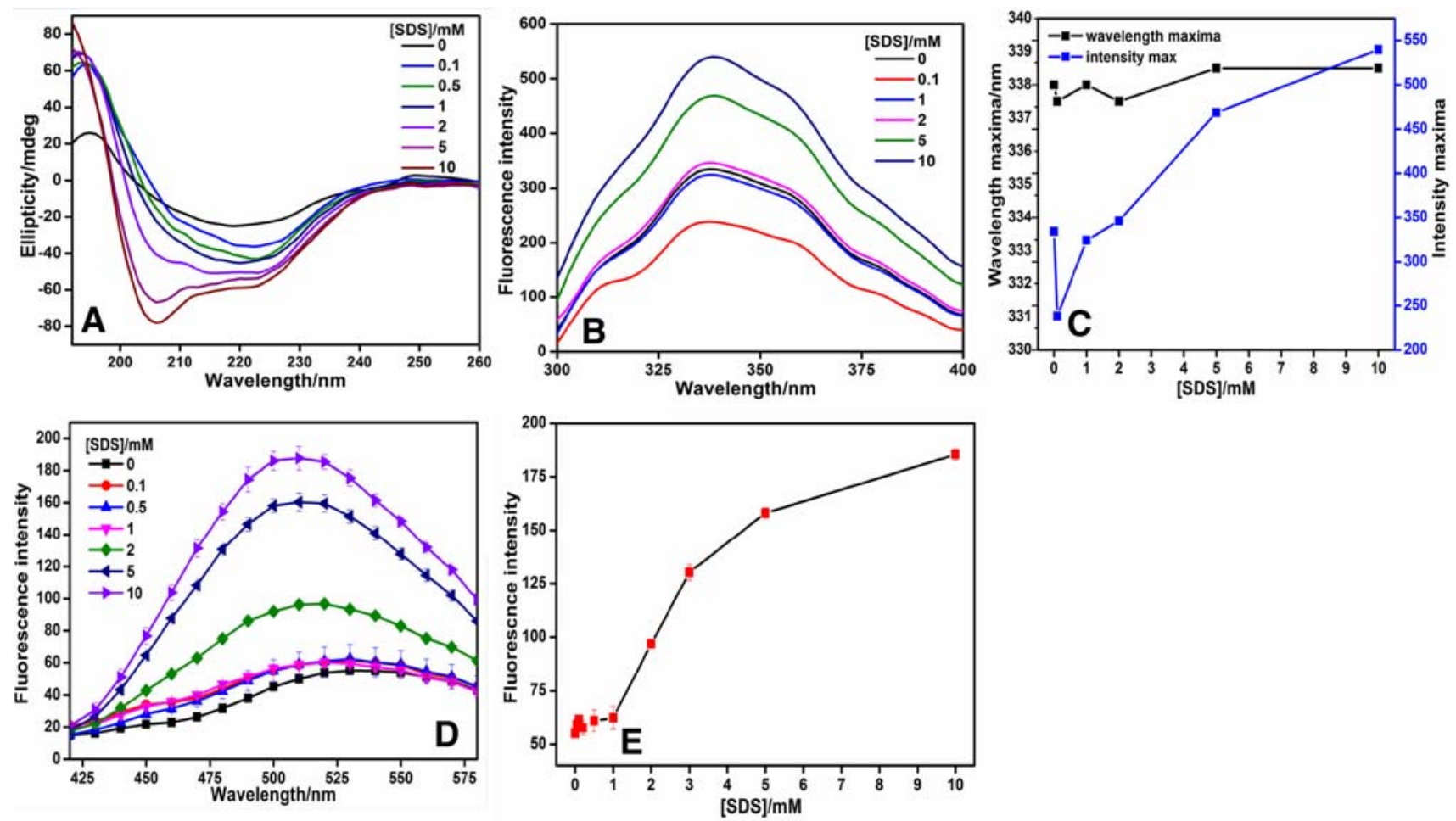

Figure 4. Conformational changes in ConA at different SDS concentrations. (A) far-UV CD spectra from 195 to 260 $\mathrm{nm}$ in presence of different SDS concentration; (B) tryptophan fluorescence emission spectra from $300 \mathrm{~nm}$ to $400 \mathrm{~nm}$ in presence of different SDS concentration; (C) intensity and wavelength maxima of tryptophan fluorescence plotted against SDS concentration; (D) ANS fluorescence spectra from $420 \mathrm{~nm}$ to $580 \mathrm{~nm}$ upon binding to ConA; and (E) change in ConA-bound ANS fluorescence intensity plotted against different SDS concentrations (each represented data wis an average of three scans with scan speed of $100 \mathrm{~nm} / \mathrm{min}$ for both $\mathrm{CD}$ and fluorescence measurements).

ConA, and insignificant changes in the secondary structure. The increase in ellipticity on SDS binding to proteins is also reported in other studies. ${ }^{40,41}$ However, above $2 \mathrm{mM}$ of SDS, a shift in trough towards lower wavelength was observed. The ellipticity minimum shifted from $222 \mathrm{~nm}$ to $208 \mathrm{~nm}$ and $206 \mathrm{~nm}$ at 5 and $10 \mathrm{mM}$ SDS, respectively (Figure 4A). The shift is also accompanied by a two-fold increase in ellipticity at $208 \mathrm{~nm}$, indicating introduction of $\alpha$-helical content in ConA. The critical micelle concentration (CMC) of SDS in $10 \mathrm{mM}$ phosphate buffer is $4.61 \pm 0.01 \mathrm{mM},{ }^{42}$ implying that below the CMC, SDS leads to minute change in the overall conformation of ConA. However, above CMC, there is a significant change in the secondary structure. At least $2 \mathrm{mM}$ SDS was required to bring significant change in the protein secondary structure. The SDS mediated denaturation of proteins thus showed presence of significant amounts of $\alpha$-helical intermediates. This particular process where proteins rearrange into predominantly all- $\alpha$ protein, following SDS-mediated denaturation, is called as constructive denaturation. ${ }^{43}$

Tryptophan fluorescence showed an increase in quantum yield with increasing SDS concentration, with maximum change at $10 \mathrm{mM}$ SDS depicting a change in the microenvironment of tryptophan residues. However, the emission maximum of tryptophan showed insignificant shift with increase in SDS concentration. The increase in quantum yield of ConA tryptophan residues upon SDS addition indicated that either ConA structure is getting compact following conformational rearrangement or the exposed tryptophan residues, $\operatorname{Trp} 88 / 182$ in native conformation, are getting buried in hydrophobic SDS micelle environment (Figures 4B and 4C). In both the cases, the orientational and vibrational entropies of tryptophan indole ring will be restricted, resulting into an enhanced quantum yield. ${ }^{44}$

When the ANS fluorescence of the SDS-ConA complexes was measured, it appeared that till $1 \mathrm{mM}$ of SDS, there was a negligible change in the fluorescence maximum depicting insignificant change in hydrophobic clusters of ConA. Concurrent with the CD spectroscopy study, it was seen that at $2 \mathrm{mM}$ SDS, there is a twofold increase in ANS quantum yield. The increase was also accompanied by a large blue shift from $530 \mathrm{~nm}$ to $515 \mathrm{~nm}$. At 5 and $10 \mathrm{mM}$ SDS, the emission maximum showed a further shift to $508 \mathrm{~nm}$ with an increase in the quantum yield. Both, rise in quantum yield and 
blue shift, indicate an increase in exposure of accessible ConA hydrophobic patches (Figures 4D and 4E). Molten globule structures and partially unfolded intermediates have high affinity for ANS. Thus, the increase in fluorescence intensity is rationalized as ANS binding to the relatively exposed ConA hydrophobic patches upon addition of SDS.

Molten globule structures are rich in secondary structures lacking important tertiary contacts. In case of ConA, interestingly, a switch from the native jelly roll motif to the all- $\alpha$ conformation is seen in the presence of SDS, which is quite evident from CD spectropolarimetry. However, the binding of ANS to ConA might be enhanced in presence of monomeric SDS which presents a hydrophobic environment below its CMC. ${ }^{45}$ The mechanism of SDS mediated protein denaturation has been contradicting. Some studies have shown that the main driving force is electrostatic interactions between the negatively charged SDS head group with the positively charged residues in proteins. ${ }^{41}$ However, another study suggests that at sub-CMC concentrations, SDS monomers bind to proteins by predominantly hydrophobic interactions causing unfolding of the tertiary structure. Whereas, above CMC, SDS micelles nucleate on the hydrophobic patches of the protein chain causing the micellar structure to expand. ${ }^{45}$ The expansion of micellar structures perturbs the interaction network, which is very essential to hold the native conformation. Thereby, the perturbed interaction network results in an expanded and weakened structure, resembling a molten globule state. In this context, one particular study indicated the effect of micelle shapes and ionic concentrations on protein structure. ${ }^{46}$ Thus, the SDS data altogether indicated that the ConA conformation is stable up to $2 \mathrm{mM}$ of SDS, whereas above this concentration the predominantly $\beta$-sheet protein converts into predominantly $\alpha$-helix protein.

\subsection{Guanidine hydrochloride induced conformational changes}

Guanidine hydrochloride $(\mathrm{GdnHCl})$ is considered one of the strongest denaturing agent. Hence, varying concentrations of $\mathrm{GdnHCl}$ from 0 to $4 \mathrm{M}$ were used to analyze conformational changes in ConA. The CD spectrum of native ConA showed a major trough at $222 \mathrm{~nm}$. The ellipticity, however, was reduced in presence of $\mathrm{GdnHCl}$. The change in ellipticity was drastic as the concentration of $\mathrm{GdnHCl}$ increased from $0 \mathrm{M}$ to $4 \mathrm{M}$ in the protein solution, indicating loss of secondary structures. In presence of $1 \mathrm{M} \mathrm{GdnHCl}$, though the minimum was retained at $222 \mathrm{~nm}$, however, a shoulder at $210 \mathrm{~nm}$ appeared, indicating the native $\beta$-sheet fold with some degree of unfolding. Additionally, in presence of $2 \mathrm{M}$ $\mathrm{GdnHCl}$, the minimum shifted to $212 \mathrm{~nm}$ that is the indication of native $\beta$-sheet in proteins. Moreover, in presence of $3 \mathrm{M} \mathrm{GdnHCl}$, there is a significant reduction in the ellipticity with a shoulder at $212 \mathrm{~nm}$, indicating loss of secondary structure with traces of native $\beta$-sheets. ${ }^{40}$ Subsequently, at $4 \mathrm{M} \mathrm{GdnHCl}$, the ellipticity was further reduced without significant change in the minima, which indicates complete unfolding of ConA into predominantly irregular secondary structure (Figure $5 \mathrm{~A}$ ).

Maximum change in ellipticity was observed at 222 $\mathrm{nm}$ (inset of Figure 5A). Thus, the extent of denaturation was expressed in terms of fraction folded $(\alpha)$ by evaluating the ellipticity at $222 \mathrm{~nm}$ at any given $\left(i^{\text {th }}\right)$ concentration of $\mathrm{GdnHCl}$, which can be calculated from the equation 1 .

$\alpha=\left([\theta]_{\mathrm{i}}-[\theta]_{\mathrm{u}}\right) /\left([\theta]_{\mathrm{f}}-[\theta]_{\mathrm{u}}\right)$

Here, $[\theta]_{\mathrm{i}}$ is the ellipticity at any particular $\left(\mathrm{i}^{\text {th }}\right)$ concentration of $\mathrm{GdnHCl},[\theta]_{\mathrm{u}}$ is the ellipticity where the protein is completely unfolded, like here, at $4 \mathrm{M}$ $\mathrm{GdnHCl}$, and $[\theta]_{\mathrm{f}}$ is the ellipticity, when the protein is fully folded, i.e., in the absence of $\mathrm{GdnHCl},{ }^{47}$ and the fraction of denatured protein is $(1-\alpha)$.

Tryptophan fluorescence studies indicated that, up to $1 \mathrm{M} \mathrm{GdnHCl}$, there is an insignificant change in the quantum yield and emission maximum. This finding shows that, although there might be slight perturbations in the overall structure of ConA as observed using CD, insignificant change in local chemical environment of tryptophan residues takes place. However, increasing $\mathrm{GdnHCl}$ concentration to $4 \mathrm{M}$ leads to an increase in quantum yield with a significant red shift in the emission maximum from $336 \mathrm{~nm}$ to $358 \mathrm{~nm}$. The red shift is accountable for exposure of tryptophan residues to more polar environment, which could be due to the unfolding of ConA (Figures 5B and 5C). The fraction of protein in the denatured state was calculated from the $\mathrm{I}_{0}$ (fluorescence intensity without denaturant), $\mathrm{I}_{\max }$ (Fluorescence intensity at maximum denaturant concentration) and I (fluorescence intensity at a particular denaturant concentration).

$\mathrm{F}_{\mathrm{d}}=\left(\left[\mathrm{I}-\mathrm{I}_{0}\right] /\left[\mathrm{I}_{\max }-\mathrm{I}_{0}\right]\right)$

Both CD and fluorescence data support the presence of intermediates from 1-2 $\mathrm{M} \mathrm{GdnHCl}$ concentrations, which could either be structured or unstructured monomers, those which are populating ahead of complete unfolding (Figure 5D).

Additionally, the ANS data were also concurrent with $\mathrm{CD}$ and fluorescence studies. It is seen that the ANS fluorescence maximum, in the absence of $\mathrm{GdnHCl}$, 

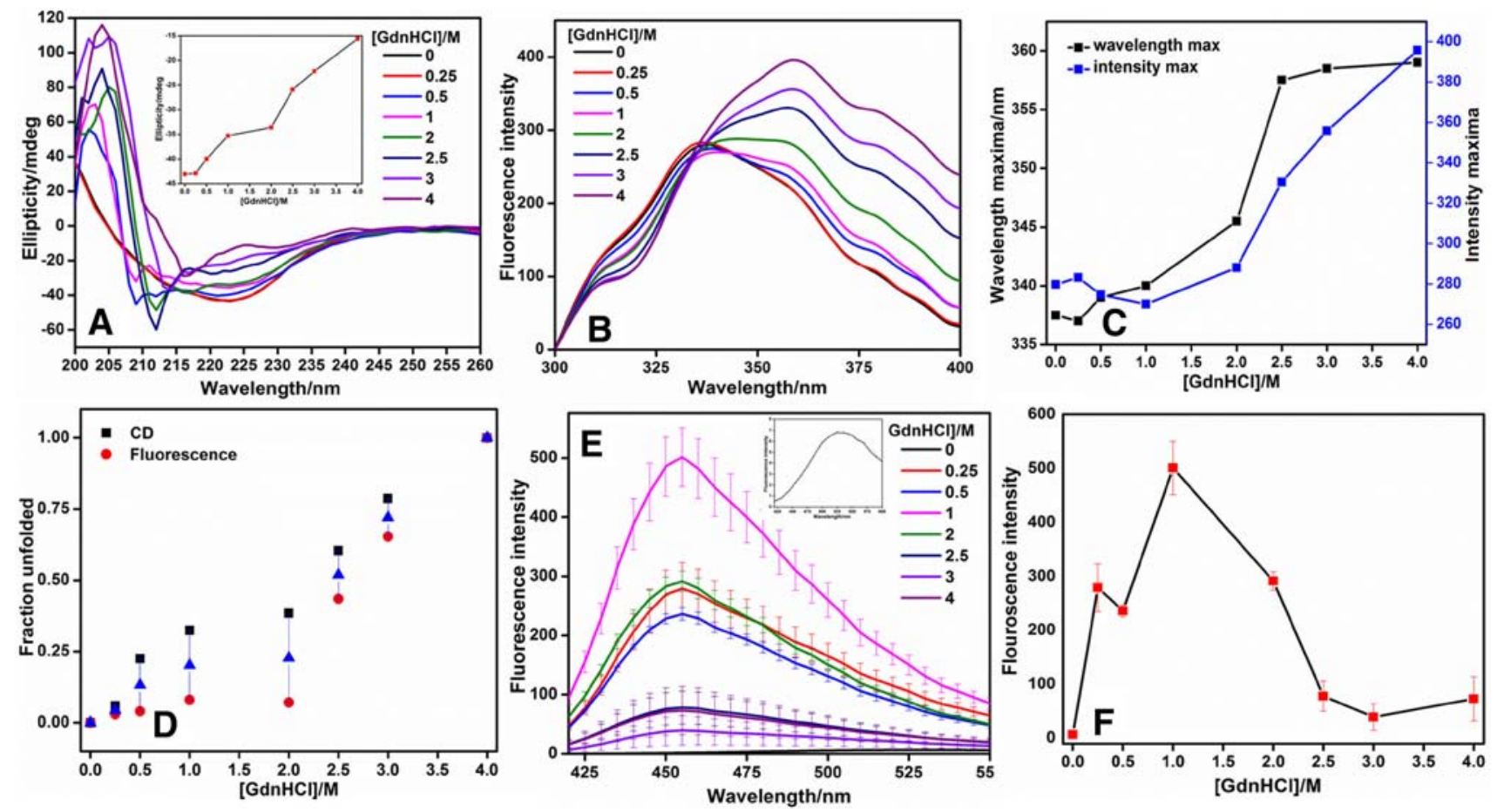

Figure 5. Conformational changes in ConA at different $\mathrm{GdnHCl}$ concentrations. (A) far-UV CD spectra from $200 \mathrm{~nm}$ to $260 \mathrm{~nm}$ and inset shows the change in ellipticity at $222 \mathrm{~nm}$; (B) tryptophan fluorescence emission spectra from $300 \mathrm{~nm}$ to $400 \mathrm{~nm}$; (C) intensity and wavelength maxima of tryptophan fluorescence plotted against $\mathrm{GdnHCl}$ concentration; (D) fraction of unfolded protein, calculated from $\mathrm{CD}$ and intrinsic fluorescence experiments, are plotted against change in $\mathrm{GdnHCl}$ concentration; (E) ANS fluorescence spectra from $420 \mathrm{~nm}$ to $580 \mathrm{~nm}$ upon binding to ConA and the inset shows ConA bound ANS fluorescence emission spectrum without $\mathrm{GdnHCl}$ in medium; and (F) ConA-bound ANS fluorescence intensity plotted against $\mathrm{GdnHCl}$ (each represented data is an average of three scans with scan speed of $100 \mathrm{~nm} / \mathrm{min}$ for both the $\mathrm{CD}$ and fluorescence measurements).

upon binding to ConA is observed at $520 \mathrm{~nm}$, depicting inaccessible hydrophobic patches at ConA surface. However, the maximum shifts to $470 \mathrm{~nm}$ in presence of $\mathrm{GdnHCl}$. At lower $\mathrm{GdnHCl}$ concentration, upto $1 \mathrm{M}$, there was an increase in tryptophan quantum yield, whereas exponential decrease in the intensity was observed as the concentration increased to $4 \mathrm{M} \mathrm{GdnHCl}$ (Figures 5E and 5F). A rise in the fluorescence intensity suggests that the accessible hydrophobic patches appear in presence of $1 \mathrm{M} \mathrm{GdnHCl}$ in ConA. Thus, the increase in ANS binding indicate the presence of molten globule like intermediates between 1-2 M GdnHCl. Whereas, lower fluorescence intensities at higher $\mathrm{GdnHCl},>2$ $\mathrm{M}$, indicate the complete unfolding of ConA, which resulted in dissolution of the hydrophobic patches.

Previously, ConA was reported to undergo a three state unfolding mechanism, induced by urea and $\mathrm{GdnHCl}$, i.e., tetramer follwed by structured monomers to unfolded monomer. It was shown that the free energy preference of quaternary tetrameric structure was greater than that of the tertiary monomeric structure, owing to structural stability of ConA by subunit association. ${ }^{31}$ While, the lower concentration of $\mathrm{GdnHCl},<1 \mathrm{M}$, helps in stabi- lizing protein structure, as also observed with ConA. ${ }^{4}$ The higher concentrations of the chaotrope completely unfolds the protein into all-irregular conformation. For ConA, $\mathrm{GdnHCl}$ data altogether, indicate the presence of molten globules between 1-2 M GdnHCl, beyond which the protein completely unfolds.

The study here indicates that ConA is a relatively very stable protein, which undergoes a multi-state unfolding mechanism, instead of two-state unfolding mechanism. The protein is completely stable for the studied $\mathrm{pH}$ range, with denaturation of lectin specific jelly roll motif into native $\beta$-sheet-like conformation at very alkaline $\mathrm{pH},>9$ (Figure 6). Interestingly, SDS concentration, above $2 \mathrm{mM}$, triggers a conformational rearrangement in ConA, from an all- $\beta$ sheet conformation to predominantly $\alpha$-helix conformation (Figure 6). However, in presence of $\mathrm{GdnHCl}$, ConA is unfolded differentially. At concentration $<3 \mathrm{M} \mathrm{GdnHCl}$, populations with jelly roll motif and native $\beta$-sheet conformations coexist, possibly with molten globule-like conformation between 1-2 M GdnHCl. However, at higher $\mathrm{GdnHCl}$ concentrations $(>3 \mathrm{M})$, complete unfolding of ConA into all-irregular conformation was observed (Figure 6). 


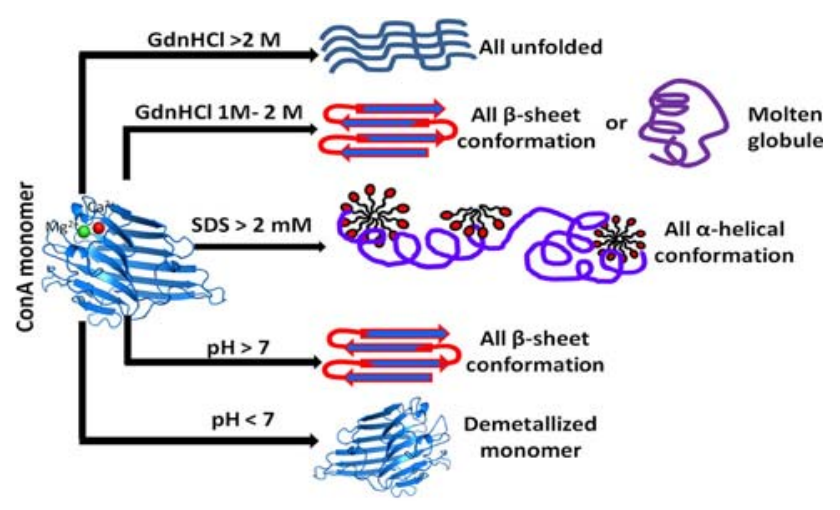

Figure 6. Schematic diagram depicting the conformational changes in ConA in different stress conditions. Guanidine hydrochloride shows complete unfolding of ConA above $2 \mathrm{M}$ concentration, whereas between 1-2 $\mathrm{M} \mathrm{GdnHCl}$ triggered the molten globule-like conformation. At basic $\mathrm{pH}$, ConA adopts all $\beta$-sheet conformation, and at acidic $\mathrm{pH}$ it retained demetallized monomeric jelly roll motif. Additionally, in presence of $>2 \mathrm{mM}$ SDS, ConA undergoes conformational rearrangement into a predominantly $\alpha$-helix conformation, rather a lectin-like all $\beta$-sheet conformation at lower concentrations of SDS.

Hence, the study indicates that ConA denaturation follows different denaturation pathways in presence of different chaotropic conditions.

\section{Conclusions}

In spite of many studies on the structural features of ConA, some key features regarding its precise denaturation pathways are missing. Our study presents ConA conformational transitions in different chaotropic conditions, like extreme $\mathrm{pH}$, different conentrations of $\mathrm{GdnHCl}$ and SDS in equilibirium conditions. We chracterized the ConA denaturation using Circular Dichroism spectroscopy, tryptophan fluorescence and ANS fuorescence. $\mathrm{GdnHCl}$ leads to initial formation of momomers followed by complete unfolding of ConA. SDS, however, could not unfold the protein completely, but resulted in conformational rearrangement into a predominantly $\alpha$-helix conformation. The effect of basic and acidic $\mathrm{pH}$ are different on ConA. ConA conformation remains significantly unperturbed at extremely low $\mathrm{pH}$, whereas extremely basic $\mathrm{pH}$ resulted in all atypical $\beta$-sheet conformation. Thus, this work indicated that ConA follows different denaturation pathways when exposed to different types of chemical and physical conditions and that, denaturation of a protein is a rather complex process than just being folding in reverse.

\section{Supplementary Information (SI)}

SDS-PAGE (10\%) run of purified ConA (Figure S1) and near UV CD spectra of ConA in presence of increasing concentration of $\mathrm{GdnHCl}$ (Figure S2) are available as Supplementary Information at www.ias.ac.in/chemsci.

\section{Acknowledgements}

We are thankful to Department of Chemistry for providing the circular dichroism facility. For the financial support, we would like to acknowledge the Department of Science and Technology, Department of Biotechnology and Ministry of Human Resource and Development, Govt. of India.

\section{References}

1. Go N 1983 Theoretical studies of protein folding Annu. Rev. Biophys. Bioeng. 12183

2. Freire E, Schon A, Hutchins B M and Brown R K 2013 Chemical denaturation as a tool in the formulation optimization of biologics Drug Discov. Today 181007

3. Makhatadze G I and Privalov P L 1992 Protein interactions with urea and guanidinium chloride: a calorimetric study J. Mol. Biol. 226491

4. Bhuyan A K 2002 Protein stabilization by urea and guanidine hydrochloride Biochemistry 4113386

5. Manning M and Colon W 2004 Structural basis of protein kinetic stability: resistance to sodium dodecyl sulfate suggests a central role for rigidity and a bias toward betasheet structure Biochemistry $\mathbf{4 3} 11248$

6. Relkin P 1994 Differential scanning calorimetry: a useful tool for studying protein denaturation Thermochim. Acta 246371

7. Becker J W, Reeke G N, Wang J L, Cunningham B A and Edelman G M 1975 The covalent and threedimensional structure of Concanavalin A. III. Structure of the monomer and its interactions with metals and saccharides J. Biol. Chem. 2501513

8. Przytycka T, Srinivasan R and Rose G D 2002 Recursive domains in proteins Protein Sci.11 409

9. Brinda K V, Mitra N, Surolia A and Vishveshwara S 2004 Determinants of quaternary association in legume lectins Protein Sci. 131735

10. Vijayan M and Chandra N 1999 Lectins Curr. Opin. Struct. Biol. 9707

11. Loris R, Hamelryck T, Bouckaert J and Wyns L 1998 Legume lectin structure Bba-Protein Struct. M. 13839

12. Reeke G, Becker J and Edelman G 1975 The covalent and three-dimensional structure of Concanavalin A. IV. Atomic coordinates, hydrogen bonding, and quaternary structure J. Biol. Chem. 2501525

13. Mitra N, Srinivas V R, Ramya T N, Ahmad N, Reddy G B and Surolia A 2002 Conformational stability of legume lectins reflect their different modes of quaternary association: solvent denaturation studies on Concanavalin A and winged bean acidic agglutinin Biochemistry 419256

14. Sinha S and Surolia A 2005 Oligomerization endows enormous stability to soybean agglutinin: a comparison of the stability of monomer and tetramer of soybean agglutinin Biophys. J. 884243 
15. Sinha S, Mitra N, Kumar G, Bajaj K and Surolia A 2005 Unfolding studies on soybean agglutinin and Concanavalin A tetramers: a comparative account Biophys. J. 881300

16. Lee Y C, Block G, Chen H, Folch-Puy E, Foronjy R, Jalili R, Jendresen C B, Kimura M, Kraft E, Lindemose $\mathrm{S}$ and $\mathrm{Lu} \mathrm{J} 2008$ One-step isolation of plasma membrane proteins using magnetic beads with immobilized Concanavalin A Protein Expr. Purif. 62223

17. H Bittiger and H P Schnebli (Eds.) 1976 Concanavalin A as a Tool (London and New York: John Wiley)

18. Ohyama Y, Kasai K i, Nomoto H and Inoue Y 1985 Frontal affinity chromatography of ovalbumin glycoasparagines on a Concanavalin A-sepharose column. A quantitative study of the binding specificity of the lectin J. Biol. Chem. 2606882

19. Pratt J, Roy R and Annabi B 2012 ConcanavalinA-induced autophagy biomarkers requires membrane type-1 matrix metalloproteinase intracellular signaling in glioblastoma cells Glycobiology 221245

20. Diwu Z, Lu Y, Zhang C, Klaubert D H and Haugland R P 1997 Fluorescent molecular probes II. The synthesis, spectral properties and use of fluorescent solvatochromic dapoxyl dyes Photochem. Photobiol. 66424

21. Agrawal B and Goldstein I J 1967 Protein-carbohydrate interaction: VI. Isolation of Concanavalin A by specific adsorption on cross-linked dextran gels BBA Protein Struct. 147262

22. Bradford M M 1976 A rapid and sensitive method for the quantitation of microgram quantities of protein utilizing the principle of protein-dye binding Anal. Biochem. 72 248

23. Kong J and Yu S 2007 Fourier transform infrared spectroscopic analysis of protein secondary structures $A B B S$ 39549

24. Vivian J T and Callis P R 2001 Mechanisms of tryptophan fluorescence shifts in proteins Biophys. J. 802093

25. Yang J T 1986 Calculation of protein conformation from circular dichroism Methods Enzymol. 130208

26. Hawe A, Sutter M. and Jiskoot W. 2008 Extrinsic fluorescent dyes as tools for protein characterization Pharm. Res. 251487

27. Goto Y and Fink A L 1989 Conformational states in. beta.-lactamase: molten-globule states at acidic and alkaline $\mathrm{pH}$ with high salt Biochemistry 28945

28. Semisotnov G V, Rodionova N A, Razgulyaev O I, Uversky V N, Gripas A F and Gilmanshin R I 1991 Study of the "molten globule" intermediate state in protein folding by a hydrophobic fluorescent probe Biopolymers 31 119

29. Ghosh G and Mandal D K 2012 Differing structural characteristics of molten globule intermediate of peanut lectin in urea and guanidine-HCl Int. J. Biol. Macromol. 51188

30. Pflumm M N and Beychok S 1974 Alkali and urea induced conformation changes in Concanavalin A Biochemistry 134982
31. Chatterjee A and Mandal D K 2003 Denaturant-induced equilibrium unfolding of Concanavalin $\mathrm{A}$ is expressed by a three-state mechanism and provides an estimate of its protein stability Biochim. Biophys. Acta Proteins Proteom. 1648174

32. Arrondo J L, Young N M and Mantsch H H 1988 The solution structure of Concanavalin A probed by FT-IR spectroscopy Bba-Protein Struct. M. 952261

33. Jha S, Sellin D, Seidel R and Winter R 2009 Amyloidogenic propensities and conformational properties of ProIAPP and IAPP in the presence of lipid bilayer membranes J. Mol. Biol. 389907

34. Zand R, Agrawal B and Goldstein I $1971 \mathrm{pH}$-dependent conformational changes of Concanavalin A Proc. Natl. Acad. Sci. 682173

35. Auer H E and Schilz T $1984 \mathrm{pH}$-dependent changes in properties of Concanavalin A in the acid $\mathrm{pH}$ range Int. $J$. Pept. Protein Res. 24462

36. Vetri V, Canale C, Relini A, Librizzi F, Militello V, Gliozzi A and Leone M 2007 Amyloid fibrils formation and amorphous aggregation in Concanavalin A Biophys. Chem. 125184

37. Royer C A 2006 Probing protein folding and conformational transitions with fluorescence Chem. Rev. 1061769

38. Ghisaidoobe A B and Chung S J 2014 Intrinsic tryptophan fluorescence in the detection and analysis of proteins: A focus on Förster resonance energy transfer techniques Int. J. Mol. Sci 1522518

39. Reynolds J A and Tanford C 1970 The gross conformation of protein-sodium dodecyl sulfate complexes J. Biol. Chem. 2455161

40. Greenfield N J 1999 Applications of circular dichroism in protein and peptide analysis TrAC, Trends Anal. Chem. 18236

41. Nielsen M M, Andersen K K, Westh P and Otzen D E 2007 Unfolding of $\beta$-sheet proteins in SDS Biophys. $J$. 923674

42. Fuguet E, Rafols C, Roses M and Bosch E 2005 Critical micelle concentration of surfactants in aqueous buffered and unbuffered systems Anal. Chim. Acta 54895

43. Jirgensons B 1982 Factors determining the reconstructive denaturation of proteins in sodium dodecyl sulfate solutions. Further circular dichroism studies on structural reorganization of seven proteins J. Protein Chem. 171

44. Vallée-Bélisle A and Michnick S W 2007 Multiple tryptophan probes reveal that ubiquitin folds via a late misfolded intermediate J. Mol. Biol. 374791

45. Bhuyan A K 2010 On the mechanism of SDS-induced protein denaturation Biopolymers $\mathbf{9 3} 186$

46. Otzen D E 2002 Protein unfolding in detergents: effect of micelle structure, ionic strength, $\mathrm{pH}$, and temperature Biophys. J. 832219

47. Greenfield N J 2006 Determination of the folding of proteins as a function of denaturants, osmolytes or ligands using circular dichroism Nat. Protoc. 12733 